\title{
Utilizando Design Science na criação de um artefato para elicitação de requisitos para jogos educacionais digitais
}

\author{
Jorge Augusto P. Silva ${ }^{1}$, Leonardo Leite R. Pimentel ${ }^{1}$, Anna Beatriz S. Marques ${ }^{2}$ \\ ${ }^{1}$ Uninorte Laureate Universities \\ Manaus - Amazonas - Brasil \\ ${ }^{2}$ Universidade Federal do Ceará \\ Russas - Ceará - Brasil \\ \{jorge.augusto.peres, leite.leonardo.rangel\}@outlook.com, \\ beatriz.marques@ufc.br
}

\begin{abstract}
Educational games are great tools used in the classroom for promoting diverse learning experiences. In the educational games development, it is necessary to know the students profile and also their needs. The existing processes for game development do not elicit both the profiles and the students' needs. This research aims to create an artifact that supports the elicitation of requirements for the educational game development. For this, we adopted the Design Science methodology. Thus, an artifact based on questionnaire and personas technique was developed. We conducted an evaluation to investigate the effectiveness of the artifact. The results demonstrate that based on the artifact, it is possible to identify functional and non-functional requirements for educational games.
\end{abstract}

Resumo. Jogos educacionais são ótimas ferramentas usadas em sala de aula, promovendo aos estudantes diversas experiências de aprendizado. No desenvolvimento de jogos educacionais é necessário conhecer o perfil dos estudantes e suas necessidades. Os processos existentes para o desenvolvimento de jogos não revelam ao mesmo tempo os perfis e necessidades dos estudantes. O objetivo desse trabalho é a criação de um artefato que apoie a elicitação de requisitos para o desenvolvimento de um jogo educacional. Para este fim, foi adotada a metodologia de Design Science. Assim, foi elaborado um artefato baseado em questionário e na técnica de personas. Uma avaliação foi conduzida com intuito de investigar a eficácia do artefato. Os resultados demonstraram que com base no artefato, é possivel identificar requisitos funcionais e não-funcionais para jogos educacionais.

\section{Introdução}

$\mathrm{Na}$ busca de tornar o processo de ensino e aprendizagem mais atrativo e eficaz para os estudantes, diversas formas de ensino têm sido exploradas. Cada vez mais, jogos educacionais têm sido elaborados e se mostrado uma técnica potencialmente eficaz de aprendizado [Shukor et al. 2015]. Segundo Monsalve et al. (2013), jogos são importantes ferramentas para apoio ao ensino, pois permitem treinar, ensinar, aprender e identificar elementos que não são comuns no modelo tradicional. Araújo et al. (2016) reforçam que o processo de desenvolvimento requer certos cuidados, além de dispor no software os conteúdos embasados por teorias de aprendizagem, deve-se enfatizar 
VII Congresso Brasileiro de Informática na Educação (CBIE 2018)

Anais do XXIX Simpósio Brasileiro de Informática na Educação (SBIE 2018)

também as dimensões técnicas. Para isso, é utilizado o design instrucional, que por sua vez, aborda desde a análise dos objetivos e necessidades de aprendizagem, até a criação de materiais, atividades e avaliação de um projeto instrucional [Savi et al. 2011].

Alguns processos foram propostos para desenvolvimento de jogos, tal como o processo ENgAGED (EducatioNAl GamEs Development), formado por características do design instrucional, contemplando a concepção do conteúdo instrucional, o design de jogos e a avaliação pelos estudantes de maneira sistemática [Battistella e Wangenheim 2016]. O processo permite caracterizar o perfil dos usuários, auxiliando a elicitação de requisitos não-funcionais. Entretanto, há a necessidade de o processo reconhecer os requisitos funcionais, que demonstram as necessidades dos usuários em termos de funcionalidades.

A organização dos recursos pedagógicos e dos conteúdos a serem estudados pode influenciar na forma com que o estudante assimilará determinado saber, podendo essa aprendizagem ocorrer mais facilmente quando essa organização ocorre de acordo com as características do estudante [Falcade et al. 2016]. Considerando a necessidade de elicitar requisitos funcionais para jogos educacionais, este trabalho objetivou desenvolver um artefato que auxilie na captura de informações para especificação de requisitos funcionais para jogos educaciona is digita is.

Para a criação do artefato, a metodologia Design Science foi adotada, considerando que esta metodologia visa a construção de artefatos para solucionar problemas do mundo real [Wieringa, 2009]. Como base para a criação do artefato, foram utilizados o questionário de aprendizes definido no processo ENgAGED e a técnica de personas PATHY que auxilia na identificação de características, necessidades e objetivos do usuário [Ferreira et al. 2015].

$\mathrm{O}$ restante deste artigo está organizado como segue: $\mathrm{Na}$ Seção 2, apresenta-se a fundamentação teórica, discorrendo sobre a elicitação de requisitos no desenvolvimento de jogos educacionais, o processo ENgAGED e a técnica PATHY. Na Seção 3, a metodologia adotada neste trabalho é apresentada e na Seção 4, o desenvolvimento do artefato é relatado. A Seção 5 descreve a validação do artefato e os resultados obtidos. E na Seção 6, finalizamos com as considerações e finais e trabalhos futuros.

\section{Fundamentação Teórica e Trabalhos Relacionados}

Esta seção aborda como a elicitação de requisitos é considerada em processos de desenvolvimento de jogos educacionais, com foco no processo ENgAGED proposto por Battistella e Wangenheim (2016). Também é apresentada a técnica de Personas Empathy (PATHY) proposto por Ferreira et al. (2015).

\subsection{Elicitação de Requisitos no Desenvolvimento de Jogos Educacionais}

A elicitação de requisitos é considerada como a parte mais crítica no desenvolvimento de software, visto que a qualidade do produto final depende fortemente da corretude dos requisitos elicitados [Ferguson e Lami 2006]. Contudo, alguns processos para desenvolvimento de jogos educacionais não possuem claramente uma fase para elicitação de requisitos. No processo de Kirkley et al. (2005), existe uma fase para análise, na qual é definida a teoria instrucional que será aplicada ao jogo, o público alvo para o qual o jogo será desenvolvido e dados adicionais que possam auxiliar no desenvolvimento do jogo. Entretanto, não fica clara a existência da coleta de 
VII Congresso Brasileiro de Informática na Educação (CBIE 2018)

Anais do XXIX Simpósio Brasileiro de Informática na Educação (SBIE 2018)

informações do público-alvo para identificar as preferências de jogos ou as dificuldades de aprendizado na disciplina.

Por sua vez o processo de Sommeregger e Kellner (2012) não possui uma fase para análise e sim uma etapa denominada projeto conceitual. Nessa fase é definido o público-alvo para o qual será direcionado o jogo bem como o tópico do jogo. Ao término dessas etapas, é realizada uma análise do público-alvo, estabelecendo a metodologia aplicada no jogo e seu grau de dificuldade. Contudo, não fica claro como são definidas as funcionalidades que o jogo deve possuir.

O processo ENgAGED foi criado para ser aplicado no contexto de instituições de ensino superior que não possuem equipes especializadas para o desenvolvimento de jogos, sendo assim um auxílio para o instrutor que queira junto aos seus estudantes desenvolver um jogo educacional [Battistella e Wangenheim, 2016]. A fase de análise do processo tem por objetivo executar as atividades de especificação da unidade instrucional, análise de aprendizes e os conceitos que levarão os estudantes ao objetivo. Em uma das tarefas desta fase caracteriza-se o público-alvo (estudantes da disciplina). Tipicamente a caracterização inclui o levantamento das seguintes informações: a faixa etária, preferências de gênero de jogos, a preferência de plataformas de jogo, modo de interação dos jogos, a frequência em que os estudantes jogam jogos digitais e não digitais, jogos favoritos e os cenários preferidos de jogos. Para esse levantamento é feito o uso do Questionário de Caracterização de Aprendizes, este questionário contém perguntas pré-determinadas pelos autores. Uma limitação do questionário é que as perguntas presentes não ajudam na elaboração do conteúdo do jogo, sendo utilizado para levantar características do jogo e informações dos aprendizes, deixando a tarefa de definição do conteúdo da estratégia instrucional a cargo do criador do jogo e não de uma análise baseada nos usuários.

\subsection{Personas e a Técnica PATHY}

Personas são arquétipos, personagens ficcionais, concebidos a partir da síntese de comportamentos observados entre consumidores com perfis extremos [Castro 2008]. Representam as motivações, desejos, expectativas e necessidades, reunindo características significativas de um grupo mais abrangente [Vianna et al. 2012]. A técnica Personas faz com que equipes possam prever os usuários e suas necessidades durante $\mathrm{o}$ processo de design, ajudando-os na escolha de soluções eficientes e comunicando sobre os possíveis usuários as partes interessadas [Mashapa et al. 2013].

Para envolver o usuário nas fases iniciais do processo de desenvolvimento, mais especificamente na fase de elicitação de requisitos, apoiando uma melhor experiência de uso do software, Ferreira et al. (2015) propuseram a técnica Personas Empathy (PATHY). Esta técnica ajuda a identificar funcionalidades e características para uma aplicação baseando-se em problemas e necessidades dos usuários [Ferreira et al. 2015]. As personas extraídas dos resultados da aplicação da técnica auxiliam na extração dos requisitos funcionais e não-funcionais da aplicação que foi proposta.

Considerando o potencial da técnica PATHY em identificar requisitos funcionais, este trabalho propõe sua adoção para complementar a estratégia de elicitação de requisitos proposta pelo processo ENgAGED por meio da criação de um novo artefato para elicitação de requisitos. 
VII Congresso Brasileiro de Informática na Educação (CBIE 2018)

Anais do XXIX Simpósio Brasileiro de Informática na Educação (SBIE 2018)

\section{Metodologia}

Segundo Wieringa (2009), o Design Science tem por finalidade a solução de problemas relevantes do mundo real por meio da construção de artefatos que permitam investigar tais problemas, propor soluções e, assim, incrementar o conhecimento por meio da proposição - ou teste - de teorias. Por esta razão, o Design Science foi utilizado para a condução desta pesquisa. A Figura 1 ilustra as fases da metodologia adotada, resumidamente descritas em seguida. $\mathrm{O}$ foco deste artigo consiste em apresentar as fases de desenvolvimento do artefato (Fase 3) e validação do artefato no contexto área fim (Fase 5).

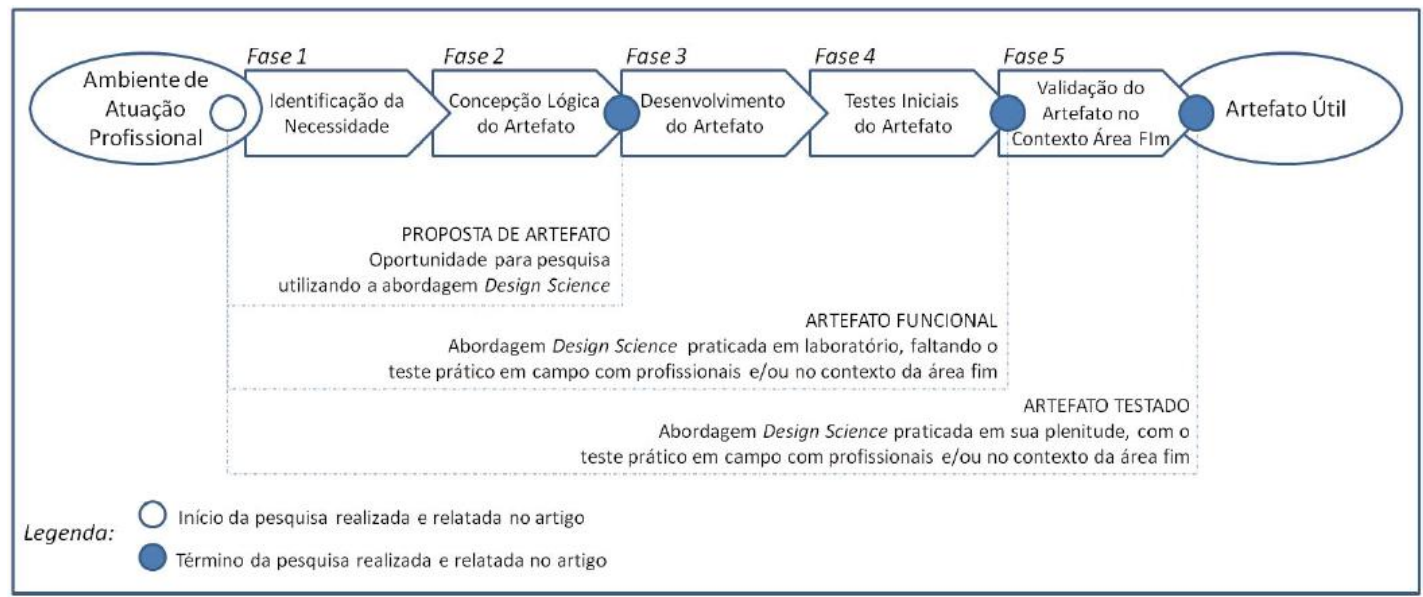

Figura 1. Fases da abordagem Design Science e os diferentes momentos do ciclo de desenvolvimento do artefato tecnológico.

Fase 1 - Identificação da Necessidade: realizou-se a revisão da literatura investigando métodos de elicitação de requisitos adotados em processos de desenvolvimento de jogos educacionais. Evidenciou-se a necessidade de criação de um artefato para o levantamento de requisitos funcionais para este tipo de aplicação.

Fase 2 - Concepção Lógica do Artefato: como base para a concepção do artefato, analisou-se o questionário proposto no processo ENgAGED e o formulário proposto na técnica PATHY, que visa a extração de requisitos funcionais de software. Selecionaram-se questões que poderiam auxiliar na coleta dos dados necessários, complementando as visões de cada técnica.

Fase 3 - Desenvolvimento do Artefato: criou-se um questionário voltado a identificar as possíveis dificuldades que os estudantes possam estar enfrentando no aprendizado de um determinado conteúdo e das características desejáveis de um jogo que aborde tal conteúdo. Criou-se também um guia com diretrizes para a extração de requisitos funcionais e não-funcionais a partir dos dados coletados por meio do artefato.

Fase 4 - Testes Iniciais do Artefato: realizou-se um teste piloto com uma pequena amostra de estudantes para que o artefato pudesse ser validado. Verificou-se também se as perguntas presentes forneceriam de fato resultados relevantes para a especificação de requisitos, a fim de eliminar perguntas que não fossem relevantes.

Fase 5 - Validação do artefato no contexto área fim: definiu-se um contexto real para validação do artefato. Então, realizou-se o planejamento, execução e análise dos resultados da validação. A análise dos resultados consistiu em verificar se o artefato criado de fato forneceria requisitos relevantes para um jogo educacional. 
VII Congresso Brasileiro de Informática na Educação (CBIE 2018)

Anais do XXIX Simpósio Brasileiro de Informática na Educação (SBIE 2018)

\section{Desenvolvimento do Artefato}

Para o desenvolvimento do artefato, inicialmente realizou-se uma análise das técnicas de elicitação selecionadas: o questionário de aprendizes do ENgAGED e a técnica PATHY, ambas baseadas em perguntas. A Tabela 1 apresenta as perguntas selecionadas para compor o novo artefato (primeira coluna) e a origem de cada pergunta (segunda coluna).

Tabela 1. Questões que compõem o novo artefato desenvolvido.

\begin{tabular}{|c|c|}
\hline Questões & Origem \\
\hline 1. Comoé sua rotina de estudo? & $\begin{array}{l}\text { PATHY: Adaptada da questão "Como é a sua } \\
\text { rotina". }\end{array}$ \\
\hline $\begin{array}{l}\text { 2. Como é o ambiente acadêmico em que } \\
\text { convive? }\end{array}$ & $\begin{array}{l}\text { PATHY: Adaptada da questão "Como é o ambiente } \\
\text { em que vive?". }\end{array}$ \\
\hline $\begin{array}{l}\text { 3. Quais dispositivos eletrônicos você utiliza em } \\
\text { geral? }\end{array}$ & $\begin{array}{l}\text { ENGAGED: Questão “Quais dis positivos } \\
\text { eletrônicos você utiliza em geral, não apenas para } \\
\text { jogo?". }\end{array}$ \\
\hline 4. Quais jogos utiliza? Com que frequência? & $\begin{array}{l}\text { PATHY: Substituído aplicativos por jogos da } \\
\text { pergunta original. }\end{array}$ \\
\hline 5. Quais jogos mais gosta, e por quê? & $\begin{array}{l}\text { PATHY: Substituído aplicativos por jogos da } \\
\text { pergunta original. }\end{array}$ \\
\hline 6. Quais jogos não gosta, e por que não? & $\begin{array}{l}\text { PATHY: Substituído aplicativos por jogos da } \\
\text { pergunta original. }\end{array}$ \\
\hline $\begin{array}{l}\text { 7. O que um jogo educacional deve ter para } \\
\text { chamar sua atenção? }\end{array}$ & $\begin{array}{l}\text { PATHY: Substituído aplicativos por jogo } \\
\text { educacional da pergunta original. }\end{array}$ \\
\hline $\begin{array}{l}\text { 8. Qual é o modo de interação preferido de } \\
\text { jogo? (Single-player; Multiplayers competitivo; } \\
\text { Multiplayers cooperativo) }\end{array}$ & $\begin{array}{l}\text { ENGAGED: Mantida pergunta original e removida } \\
\text { as opções Multigrupos competitivo e Multigrupos } \\
\text { cooperativos por não se encaixarem no escopo do } \\
\text { artefato. }\end{array}$ \\
\hline $\begin{array}{l}\text { 9. Qual é a sua preferência de gêneros de jogo? } \\
\text { (ação-aventura; adivinhação; corrida; } \\
\text { estratégia;puzzle;RPG;simulação;outro) }\end{array}$ & $\begin{array}{l}\text { ENGAGED: Mantida pergunta original e rem ovida } \\
\text { a opção Roll-and-Move, pois é um gênero de jogos } \\
\text { de tabuleiro. }\end{array}$ \\
\hline $\begin{array}{l}\text { 10. Você tipicamente prefere jogar jogos em qual } \\
\text { plataforma? (computador stand-alone; } \\
\text { computador online; console; mobile - } \\
\text { smartphone; mobile - tablete) }\end{array}$ & $\begin{array}{l}\text { ENGAGED: Mantida pergunta original sendo que } \\
\text { as opções Jogo não-digital de tabuleiro, Jogo não- } \\
\text { digital de cartas, Jogo não-digital papel \& lápis, } \\
\text { Jogo não-digital acessórios foram removidas por } \\
\text { não se encaixar no escopo do artefato. }\end{array}$ \\
\hline 11. Já utilizou algum jogo educacional? Qual? & NOVA \\
\hline $\begin{array}{l}\text { 12. Você possui algum comentário a mais para } \\
\text { fazer sobre suas preferências de jogo? }\end{array}$ & ENGAGED: Mantida pergunta original \\
\hline $\begin{array}{l}\text { 13. Com relação à disciplina <disciplina >com } \\
\text { que conteúdos anda preocupado } \\
\text { ultimamente? }\end{array}$ & $\begin{array}{l}\text { PATHY: Adaptada da questão "Com o que anda } \\
\text { preocupado ultimamente?" }\end{array}$ \\
\hline $\begin{array}{l}\text { 14. O que o frustra em relação ao aprendizado } \\
\text { dos conteúdos da disciplina < <lisciplina>? }\end{array}$ & PATHY: Adaptada da questão "O que o frustra?" \\
\hline $\begin{array}{l}\text { 15. Que problem as você enfrenta no aprendizado } \\
\text { da disciplina < disciplina>? }\end{array}$ & $\begin{array}{l}\text { PATHY: Adaptada da questão "Que problemas ele } \\
\text { enfrenta?" }\end{array}$ \\
\hline $\begin{array}{l}\text { 16. Como um jogo educacional pode ajudar a } \\
\text { resolver este problema? }\end{array}$ & $\begin{array}{l}\text { PATHY: Adaptada da questão "Como a aplicação } \\
\text { pode ajudar a resolver este problema?" }\end{array}$ \\
\hline $\begin{array}{l}\text { 17. Quais recursos um jogo educacional deveria } \\
\text { ter para auxiliar em seu problema de } \\
\text { aprendizagem? }\end{array}$ & $\begin{array}{l}\text { PATHY: Adaptada da questão "O que precisa para } \\
\text { acabar com estes problemas?" }\end{array}$ \\
\hline $\begin{array}{l}\text { 18. Você utiliza algum recurso (aplicativo/jogos } \\
\text { educacionais/site) para auxiliar nos } \\
\text { problemas enfrentados no aprendizado da } \\
\text { disciplina < disciplina>? }\end{array}$ & $\begin{array}{l}\text { PATHY: Adaptada da questão "Há algum } \\
\text { aplicativo/site que resolve estes problemas?" }\end{array}$ \\
\hline $\begin{array}{l}\text { 19. Há alguma ideia de interface que possa ser } \\
\text { reutilizada no jogo a ser desenvolvido? }\end{array}$ & $\begin{array}{l}\text { PATHY: Substituído aplicativo por jogo da } \\
\text { pergunta original. }\end{array}$ \\
\hline
\end{tabular}


VII Congresso Brasileiro de Informática na Educação (CBIE 2018)

Anais do XXIX Simpósio Brasileiro de Informática na Educação (SBIE 2018)

As técnicas possuíam algumas perguntas similares, consideradas redundantes. Nestes casos, gerava-se uma única pergunta equivalente a ambas as técnicas. Haviam ainda perguntas consideradas não relevantes para o contexto de jogos digitais, uma vez que a PATHY é proposta para o desenvolvimento de aplicativos em geral. As questões presentes na técnica PATHY foram em sua maioria adaptadas para um contexto de utilização em jogos educacionais. As questões presentes no questionário do ENgAGED referentes a características de jogos não digitais não foram consideradas na concepção do novo artefato. Para auxiliar no levantamento de requisitos a partir dos dados coletados pelo artefato proposto, elaborou-se também um guia para extração de requisitos (Tabela 2). Com o desenvolvimento do guia para extração e a condução de testes iniciais, observou-se que a questão 2 poderia ser removida.

Tabela 2. Guia para extração de cada requisito.

R001 - Plataforma do jogo

Esse requisito é extraído das questões 2. "Quais dispositivos eletrônicos você utiliza em geral?" e 8.

"Você tipicamente prefere jogar jogos em qual plataforma?", as respostas dessas questões devem s er comparadas visando atender o que a maioria dos estudantes opinou, é importante observar outras preferências dos estudantes que podem aparecer na questão 11. "Você possui algum comentário a mais para fazer sobre suas preferências de jogo?". Na escolha da plataforma deve-se considerar pelo menos, os dois dispositivos mais usados caso a diferença entre a preferência dos estudantes seja pequena, caso algum dos dispositivos tenha uma preferência muito alta (mais de $30 \%$ de diferença) este deve ser a preferência escolhida quanto à plataforma.

\section{R002 - Modo de Interação}

Os resultados deste requisito são provenientes principalmente da questão 6. "Qual é o modo de interação preferido de jogo?", porém as respostas das questões 3. "Quais jogos utiliza? Com que frequência?", 4. "Quais jogos mais gosta, e por quê?" e 5. "Quais jogos não gosta, e por que não?" também servem de base para a decisão. Caso a diferença de preferências dos estudantes seja pequena é recomendável que o jogo tenha os dois modos de interação, caso não seja possível, pode se escolher a preferência da maioria levando em conta as ressalvas que podem ser feitas nas questões 5 e 11.

\section{R003 - Gênero Desejável}

Esse requisito deve ser avaliado de acordo com as respostas obtidas das questões 3, 4, 5 e 7. Apes ar da questão 7. "Qual é a sua preferência de gêneros de jogo?" ser a questão específica para levantar a preferência de gênero de jogo, é importante observar as outras questões como os jogos que os estudantes mais gostam, os que menos gostam e os comentários.

\section{R004 - Características desejáveis}

Esse requisito é obtido com base nos comentários dos estudantes realizados durante o qu e s tion ário, principalmente os descritos nas questões 4. "Quais jogos mais gosta, e por quê?", 9. "Já utilizou algum jogo educacional? Qual?", 10. “O que um jogo educacional deve ter para chamar sua atenção?”, 11. "Você possui algum comentário a mais para fazer sobre suas preferências de jogo?" e 18. "Há al gum a ideia de interface que possa ser reutilizada no jogo a ser des envolvido?". As respostas são qualitativas e os dados devem ser analisados para extração das características, porém pode-se observar se existem tendências entre os estudantes.

R005 - Conteúdo disciplinar do Jogo

Os conteúdos devem ser extraídos pela maioria das opiniões na questão 12. "Com relação à dis ciplina < disciplina > com que conteúdos anda preocupado ultimamente?", observando os comentários dos estudantes. Pode ser utilizado mais de um conteúdo ficando essa decisão a cargo do criador do jogo. R006 - Conteúdo desejável

O conteúdo desejável consiste em como os estudantes esperam que o conteúdo disciplina seja aplicado em um jogo, como por exemplo, em que contexto o jogo ocorrerá. Esse requisito pode ser extraído ao observar as respostas das questões 13. "O que o frustra em relação ao aprendizado dos conteúdos da disciplina <disciplina>?", 14. "Que problemas você enfrenta no aprendizado da disciplina <disciplina>?", 15. "Como um jogo educacional pode ajudar a resolver este problema?", 16. “Quais recursos um jogo educacional deveria ter para auxiliar em seu problema de aprendizagem?" e 17. "Você utiliza algum recurso para auxiliar nos problemas enfrentados no aprendizado da disciplina?". Novamente as respostas são qualitativas e devem ser analisadas para extração d os requisitos. 
VII Congresso Brasileiro de Informática na Educação (CBIE 2018)

Anais do XXIX Simpósio Brasileiro de Informática na Educação (SBIE 2018)

\section{Validação do artefato no contexto área fim}

$\mathrm{O}$ artefato foi projetado para ser respondido por futuros usuários do jogo educacional e envolvê-los na fase de elicitação de requisitos do desenvolvimento do jogo. Com isso, os dados coletados podem auxiliar a entender os problemas do usuário e refletir sobre como esses problemas poderão ser resolvidos através do jogo educacional.

Os usuários que responderão ao questionário deverão receber instruções sobre o que se trata o questionário e a sua finalidade. Após o preenchimento, o questionário deverá ser recolhido para que se inicie a fase de análise de informações pelos pesquisadores. É recomendado que o artefato seja utilizado em equipe para que haja uma melhor discussão na fase de análise e especificação de requisitos.

\subsection{Planejamento e Execução}

A validação do artefato foi realizada em uma turma do $6^{\circ}$ período do curso de Bacharelado em Ciência da computação no contexto da disciplina de Engenharia de Software. Os estudantes foram instruídos pela instrutora e pelos pesquisadores sobre o objetivo do questionário. Além do questionário, cada estudante recebeu duas vias do Termo de Consentimento Livre e Esclarecido, no qual concordavam em fornecer os dados para análise e validação do artefato. Desta validação, participaram 36 estudantes sendo a sua maioria do sexo masculino (33 estudantes), com a idade entre 19 e 35 anos.

\subsection{Análise dos Resultados e Discussão}

A maioria dos estudantes respondeu que estuda diariamente pelo menos para reforçar o conteúdo passado em sala de aula, nos quais alguns utilizam recursos para seu estudo como sites, livros, artigos ou vídeos sobre os assuntos ministrados em sala de aula e outros estudantes fazem uso de algum aplicativo para auxiliar nos estudos.

Entre os dispositivos mais utilizados todos os 36 estudantes afirmaram que fazem uso de um computador, e 31 deles que fazem uso de smartphone. O console ainda é a preferência dos estudantes quanto à plataforma utilizada para jogos sendo utilizado por 18 dos estudantes. Seguindo como preferência, temos o uso de computador online escolhido por 15 estudantes e smartphone por 14 estudantes.

Entre preferências de jogos a amostra varia bastante, porém entre os jogos que costumam jogar e os jogos que mais gostam, há uma preferência principalmente pelos gêneros de ação e aventura com total de 26 dos estudantes. Foram citados vários jogos do estilo FPS como Call of Duty e Counter Strike; dentre os jogos de estratégia 25 estudantes relataram jogos como League of Legends e DotA. A razão dessa preferência se deve ao fato de os estudantes gostarem de jogos em que se sintam desafiados de alguma forma. Entre os jogos menos apreciados pelos estudantes estão principalmente os de RPG e algumas menções a jogos de estilo FPS. Em modos de interação, os estudantes ficaram divididos entre singleplayer e multiplayer competitivo com 18 respostas para cada.

Em relação aos jogos educacionais já utilizados, a maioria respondeu que já utilizou algum jogo e o mais citado foi o Duolingo. Para alguns estudantes, um jogo educacional deveria possuir mobilidade, mostrar o desempenho do estudante quanto ao progresso nas atividades, poder interagir com outros estudantes e demonstrar de forma prática o conteúdo aprendido em sala de aula. 
VII Congresso Brasileiro de Informática na Educação (CBIE 2018)

Anais do XXIX Simpósio Brasileiro de Informática na Educação (SBIE 2018)

Com relação aos conteúdos que mais enfrentam dificuldades com a disciplina, oito estudantes relataram que processos de desenvolvimento de software é o conteúdo que mais os preocupam, a mesma quantidade de estudantes afirmou que não está preocupado com nenhum conteúdo da disciplina, e seis estudantes afirmaram que estão preocupados com todo o conteúdo da disciplina. Entre os conteúdos mais específicos o mais citado foi o modelo BPMN (Business Process Modeling Notation). Alguns estudantes relataram que a falta de prática dos assuntos é o fator pelo qual eles sentem maior dificuldade de aprendizado. Como sugestão da maneira pela qual um jogo educacional possa ajudar no aprendizado, foi citado que o jogo mostrasse a aplicabilidade dos conteúdos em contextos reais e como sugestão de interface o que foi mais citado foi que seria bom algo como o aplicativo Duolingo. Por fim, com base nessas informações foram levantados os requisitos do jogo educacional, que podem ser vistos na Tabela 3.

Tabela 3. Requisitos levantados para o jogo.

\begin{tabular}{l} 
R001 - Plataforma do jogo \\
\hline Web ou PC \\
\hline $\begin{array}{l}\text { O des envolvimento para web tem preferência, pois os números de dispositivos usados frequentemente } \\
\text { ficaram divididos entre PC e smartphone. Como segunda opção a plataforma ideal é o PC já que } \\
\text { recebeu o maior número de respostas. }\end{array}$
\end{tabular}

R002 - Modo de Interação

Multiplayer

Os estudantes se mostraram bem divididos quanto ao modo de interação, então para que se tenha uma melhor experiência por parte dos usuários, o ideal seria que o modo do jogo fos se multiplayer.

R003 - Gênero Desejável

Estratégia

Os dois gêneros mais votados pelos estudantes foram ação/aventura e estratégia. Foi escolhido o gênero de estratégia, pois, analisando a pergunta "Quais jogos não gosta, e por que não?", observou-se uma rejeição ao gênero de ação/aventura.

R004 - Características desejáveis

Dificuldade elevada, Interação com outros usuários, design simples, Qualidade de conteúdo

Algumas características foram mais citadas pelos estudantes, em relação à dificuldade elevada como o objetivo do jogo é ensinar de maneira simples, o ideal seria que o jogo apresentasse uma opção para modificar a dificuldade ou ainda diferentes níveis com diferentes tipos de dificuldades.

R005 - Conteúdo disciplinar do Jogo

Processos de Desenvolvimento de software, BPMN

A maioria dos estudantes respondeu que tinha algum problema em relação aos processos de desenvolvimento, o mais citado entre eles foi o BPMN. Outros, no entanto descreveram ter problemas para diferenciar algumas das características dos processos. O ideal seria então que o conteúdo fos se sobre os processos de desenvolvimento de software evidenciando a diferença existente entre eles e com ênfase para a modelagem de processos com BPMN.

R006 - Conteúdo desejável

Simulação do conteúdo em ambientes reais

Foi descrito por alguns estudantes que seria ideal se o conteúdo vis to no jogo tivesse o contexto da aplicação no mundo real como, por exemplo, em uma equipe de desenvolvimento e que fosse visualizado os papéis de cada um dentro da equipe, ou ainda dentro de uma grande empresa de desenvolvimento de softwares. 
VII Congresso Brasileiro de Informática na Educação (CBIE 2018)

Anais do XXIX Simpósio Brasileiro de Informática na Educação (SBIE 2018)

Os requisitos iniciais descritos na Tabela 3 foram levantados reunindo as informações das perguntas do questionário respondidas pelos estudantes e com base no guia para extração de requisitos presente na Tabela 2. Os resultados demonstram que foi possível identificar requisitos funcionais e não-funcionais a partir dos dados coletados por meio do novo artefato. Por outro lado, nem todos os requisitos relacionados às características desejáveis (R004) foram identificadas pois as respostas dos estudantes foram balanceadas, sem indicar uma tendência.

Para melhor explorar a especificação de requisitos, uma análise com foco mais qualitativo deve ser conduzida sobre os dados coletados. Ainda assim, o artefato criado e o guia para extração de requisitos fornecem uma maior orientação para $o$ desenvolvimento de jogos educaciona is por equipes não especialistas em jogos.

\section{Considerações Finais e Trabalhos Futuros}

Este artigo apresentou a elaboração de um artefato para elicitação de requisitos funcionais e não-funcionais para um jogo educacional, seguindo a metodologia Design Science, que tem como objetivo a criação de artefatos para solução de problemas do cotidiano. $\mathrm{O}$ artefato é um questionário composto por perguntas selecionadas e adaptadas do questionário de aprendizes proposto pelo processo ENgAGED e da técnica PATHY. O artefato tem como objetivo capturar as necessidades/dificuldades que os estudantes possuem em uma determinada disciplina e as características para tornar o jogo educacional adequado às necessidades e preferências dos estudantes.

Após a aplicação do questionário, a análise quantitativa e qualitativa das questões respondidas foi conduzida em busca de tendências nos dados que indicassem as preferências dos estudantes. Com base no guia proposto para extração de requisitos, as tendências observadas foram utilizadas para a especificação de requisitos iniciais do jogo educacional digital. Os requisitos iniciais obtidos demonstram que o artefato criado apoia a elicitação de requisitos funcionais e não-funcionais de um jogo educacional. Contudo, nem sempre a identificação de tendências é possível. Nestes casos, é necessária a adoção de métodos de análise qualitativa sobre os dados coletados com o intuito de melhor explorar as respostas dos estudantes. Ainda assim, os requisitos iniciais obtidos podem direcionar o desenvolvimento de jogos adequados ao perfil dos estudantes e alinhados às suas necessidades em termos de aprendizado.

Como trabalhos futuros, pretende-se investigar o uso do artefato em um projeto real de desenvolvimento de jogos educacionais, com profissionais não especialistas em jogos educacionais. Assim, será possível investigar se o artefato de fato apoia a elicitação de requisitos por equipes não especialistas. Além disso, diretrizes para a extração de requisitos por meio de métodos qualitativos de análise podem ser criados, visando uma maior eficácia da especificação de requisitos funcionais. Como o foco do artefato é identificar requisitos funcionais e não-funcionais para o jogo, em trabalhos futuros também seria possível utiliza-lo em conjunto com abordagens para definição dos requisitos pedagógicos de jogos educacionais.

\section{Referências}

Battistella, P. E. e Wangenheim, C. G. von. (2016) "ENgAGED: Um Processo de Desenvolvimento de Jogos para Ensinar Computação", Anais do XXVII Simpósio Brasileiro de Informática na Educação, Uberlândia/MG, pp. 380-389. 
VII Congresso Brasileiro de Informática na Educação (CBIE 2018)

Anais do XXIX Simpósio Brasileiro de Informática na Educação (SBIE 2018)

Castro, J. W., Acunã, S. T. e Juristo, N. (2008) "Enriching requirements analysis with the personas technique", Proceedings of the International Workshop, Interplay Between Usability Evaluation And Software Development - I-USED 2008, pp.13-18.

Falcade, A., Krassmann, A. L., Freitas, V., Kautzmann, T., Jardim, R. R., Bernardi, G., e Medina, R. D. (2016). "Design Instrucional: um comparativo de metodologias para definição de abordagem em mundo virtual". Anais do XXVII Simpósio Brasileiro de Informática na Educação, Uberlândia/MG, pp. 80-89.

Ferguson, R. e Lami, G. (2006) "An Empirical Study on The Relationship Between Defective Requirements and Test Failures. Software Engineering Workshop", IEEE Computer Society, April, pp. 7-10.

Ferreira, B., Conte, T. e Barbosa S. (2015) 'Eliciting Requirements using Personas and Empathy Map to Enhance the User Experience", 29 ${ }^{\circ}$ Simpósio Brasileiro de Engenharia de Software, Belo Horizonte, pp. 80-89.

Araújo, D. C., Rodrigues, A. N., Lima, P. V. L., Ferreira, M. A. D. e Santos, H. R. M. (2016) "Processo de desenvolvimento do jogo sério Missão Aedes: relações entre objetivos pedagógicos, ludicidade e implicações de design", Anais do XXVII Simpósio Brasileiro de Informática na Educação (SBIE), p. 597.

Kirkley, S. E., Tomblin, S. e Kirkley, J. (2005) "Instructional Design Authoring Support for the Development of Serious Games and Mixed Reality Training", Interservice/Industry Training, Simulation, and Education Conference, pp. 1-11.

Mashapa, J., Chelule, E., Greunen, D. Van e Veldsman, A. (2013) 'Managing User Experience - Managing Change", In: Human - Computer Interaction - INTERACT 2013, Springer Berlin Heidelberg, pp. 660-677.

Monsalve, E., Werneck, V., e Leite, J. (2013) "Incorporando transparência na pedagogia através do uso de jogos para ensino", Anais do XXVII Simpósio Brasileiro de Engenharia de Software (SBES), Brasília, pp.75-80.

Savi, R., Wangenheim, C. G. von e Borgatto, A (2011) "Um Modelo de Avaliação de Jogos Educacionais na Engenharia de Software", Anais do XXV Simpósio Brasileiro de Engenharia de Software (SBES)/São Paulo/Brazil, p. 194.

Sommeregger, P. e Kellner, G. (2012) "Brief Guidelines for Educational Adventure Games Creation (EAGC)", Digital Game and Intelligent Toy Enhanced Learning, IEEE 4th Int. Conf., Takamatsu, Japão, pp. 120-122.

Shukor, Nurbiha A., Zaidatun Tasir, e Henny Van der Meijden (2015) "An Examination of Online Learning Effectiveness Using Data Mining", In: Procedia-Social and Behavioral Sciences 172, pp.555-562.

Vianna, M., Vianna, Y., Adler, I. K., Lucena, B., Russo, B. (2012) 'Design Thinking: Inovação em Negócios", MJV Press, p. 80.

Wieringa, R. (2009) "Design science as nested problem solving," Proc. of the 4th Int. Conf. on Design Science Research in Information Systems and Technology (DESRIST '09), ACM, pp.1-8. 